Draft Version November 23, 2021

Preprint typeset using $\mathrm{LAT}_{\mathrm{E}} \mathrm{X}$ style emulateapj v. 5/2/11

\title{
A MULTI-WAVELENGTH ANALYSIS OF NGC 4178: A BULGELESS GALAXY WITH AN AGN
}

\author{
N. J. Secrest ${ }^{1}$, S. Satyapal ${ }^{1}$, S. M. Moran ${ }^{2}$, C. C. Cheung ${ }^{3}$, M. Giroletti ${ }^{4}$, M. Gliozzi ${ }^{1}$, M. P. Bergmann $^{5}$ \& \\ A. C. $\mathrm{SeTH}^{6}$ \\ Draft version November 23, 2021
}

\begin{abstract}
We present Gemini longslit optical spectroscopy and VLA radio observations of the nuclear region of NGC 4178, a late-type bulgeless disk galaxy recently confirmed to host an AGN through infrared and X-ray observations. Our observations reveal that the dynamical center of the galaxy is coincident with the location of the Chandra X-ray point source discovered in a previous work, providing further support for the presence of an AGN. While the X-ray and IR observations provide robust evidence for an AGN, the optical spectrum shows no evidence for the AGN, underscoring the need for the penetrative power of mid-IR and X-ray observations in finding buried or weak AGNs in this class of galaxy. Finally, the upper limit to the radio flux, together with our previous X-ray and IR results, is consistent with the scenario in which NGC 4178 harbors a deeply buried AGN accreting at a high rate.

Subject headings: Galaxies: active — Galaxies: spiral — Optical: Galaxies — Radio: Galaxies — Black hole physics
\end{abstract}

\section{INTRODUCTION}

The fact that supermassive black holes (SMBHs, $\left.M_{\mathrm{BH}} \gtrsim 10^{6} \mathrm{M}_{\odot}\right)$ or intermediate-mass black holes (IMBHs, $10^{2} \mathrm{M}_{\odot} \lesssim M_{\mathrm{BH}} \lesssim 10^{6} \mathrm{M}_{\odot}$ ) can be found in late-type galaxies in which there is no evidence for classical bulge is now on firm empirical ground (Filippenko \& Ho 2003; Barth et al. 2004; Greene \& Ho 2004, 2007; Satvapal et al. 2007; Dewangen et al. 2008; Ghosh et al. 2008; Mathur et al. 2008; Satvapal et al. 2008; Shields et al. 2008; Barth et al. 2009; Desroches \& Ho 2009; Gliozzi et al. 2009; Satvapal et al. 2009; Jiang et al. 2011a.b; McAlpine et al. 2011; Secrest et al. 2012; Araya Salvo et al. 2012; Simmons et al. [2013). The presence of such black holes in these galaxies points to a new understanding of the formation mechanisms of large nuclear black holes. This is further bolstered by the remarkable evidence for the presence of an active galactic nucleus (AGN) in the dwarf galaxy Henize 2-10 (Reines et al. 2011) and the mounting evidence for AGN activity in blue compact dwarf galaxies (Izotov et al. 2007; Izotov \& Thuan 2008; Izotov et al. 2010). It is also likely that the particularly bright ultraluminous X-ray source HLX-1, also an IMBH (Soria et al. 2010; Webb et al. 2012), is the remnant of a dwarf galaxy (Farrell et al. 2012). Central to this new understanding is the black hole occupation fraction, which can provide insights into differentiating

\footnotetext{
${ }^{1}$ George Mason University, Department of Physics \& Astronomy, MS 3F3, 4400 University Drive, Fairfax, VA 22030, USA

2 Johns Hopkins University, Department of Physics \& Astronomy, 366 Bloomberg Center, 3400 N. Charles Street, Baltimore, MD 21218, USA

3 Space Science Division, Naval Research Laboratory, Washington, DC 20375-5352, USA

${ }^{4}$ INAF Istituto di Radioastronomia, 40129 Bologna, Italy

${ }^{5}$ NOAO Gemini Science Center (Chile), P.O. Box 26732, Tucson, AZ 85726

${ }^{6}$ Department of Physics and Astronomy, University of Utah, Salt Lake City, UT 84112, USA
}

whether the first 'seed' black holes in the Universe originated from Population III stars, or whether the first seed black holes had a more exotic origin, such as the hypothetical 'quasistar' direct-collapse scenario (see Bonoli et al. 2012, and references therein) that may have unfolded in the early Universe due to the extremely low metallicity environment and lack of stellar clumping (for details on how the black hole occupation fraction differs under these two scenarios, see, e.g., van Wassenhove et al. 2010). These bulgeless and dwarf galaxies are of particular importance to this question because classical bulges are thought to form through mergers, which erase information on the progenitor galaxies' black hole masses after they coalesce, while pseudobulges are thought to form through secular processes (Kormendy \& Kennicutt 2004). Nuclear black holes in these galaxies may thus be thought of as 'pristine', having likely gone through only secular growth during the extent of cosmic history, and preserving the characteristics of the seed population from which they originated. While it is not yet technically feasible to obtain an unbiased estimate of the black hole occupation fraction at masses $\lesssim 10^{6} \mathrm{M}_{\odot}$ due to limitations on detectability 7 , we can nonetheless begin to build a census of AGNs in low mass and bulgeless galaxies that will contribute to our understanding of this subject.

One such galaxy is NGC 4178, a late-type, bulgeless spiral galaxy $(d=16.8 \mathrm{Mpc})$ that was recently confirmed to host an AGN through high-resolution Chandra X-ray observations (Secrest et al. 2012, hereafter S12), with a nuclear black hole mass, inferred through mid-IR and radio/X-ray correlations, between $\sim 10^{4}-10^{5} \mathrm{M}_{\odot}$. While the presence of an AGN has been confirmed in this object, the optical confirmation of the presence of

7 Although see Greene (2012) for a first-pass estimate, which tentatively finds that the direct-collapse scenario may better explain the (limited) data we currently have on this black hole demographic. 
an AGN in NGC 3621 (Barth et al. 2009), a bulgeless spiral galaxy with highly similar X-ray/mid-IR properties, has motivated us to explore the optical properties of NGC 4178. While an optical spectrum of the nuclear region already exists (Ho et al. 1995), we have determined that it did not actually cover the region containing the nuclear X-ray source, and so it is not known how the ionization of the interstellar medium (ISM) changes near the source, if at all. Furthermore, it is not definitively known whether or not some of the $[\mathrm{Ne} \mathrm{V}]$ emission from the nucleus (Satyapal et al. 2009) originates in a starburst and/or shocks. If this were true, then it would reduce the inferred bolometric luminosity of the AGN and therefore the lower limit on the mass of the nuclear black hole. By examining the star formation properties of the nuclear region, we can explore this possibility and make the inferred bolometric luminosity more robust.

The aim of this paper is to further characterize the nuclear environment around the AGN in NGC 4178. We used Gemini longslit spectroscopy to derive the dynamical center of the galaxy, explore the ionization state of the circumnuclear ISM, and explore the presence of star formation. We also obtained new Very Large Array (VLA) observations to further constrain the mass of the nuclear black hole. In $\S 2$, we describe the Gemini observations and data reduction, as well as the new VLA data. We continue with a description of our results in $\S 3$, before discussing the implications of our results in $\S 4$. We give a summary and our primary conclusions in $\S 5$.

\section{OBSERVATIONS AND DATA REDUCTION}

\subsection{Gemini Spectroscopic Observations}

NGC 4178 was observed with the Gemini GMOS-N, long-slit (1" $)$, B600-G5307 grating on 2012 February 14th and 21st for 3600 seconds and 2400 seconds (1200 seconds per frame), respectively (Program ID: GN-2012AQ-14) 8 . The position angle of the slit was $25^{\circ}$ east of north so as to align the slit along the major axis of the galaxy. The effective airmass for the observations ranged from 1.016 to 1.041, making differential refraction effects negligible. Upon inspection of the pointing frames, it became apparent that the seeing was considerably worse on the second night and the pointing missed the nucleus. Consequently, we carried out our primary analysis on the three successful observations from the first night.

The data were reduced and calibrated with the Gemini package for IRAF, v. 1.11, following standard procedures. Cosmic rays were cleaned using the gscrspec multiextension fits wrapper for L.A.Cosmic van Dokkum 2001) and using the default parameters. Despite the large $\left(\sim 330^{\prime \prime}\right)$ length of the slit, some contamination remained from the galaxy even at the ends. While this was a minor drawback and did not affect the subsequent analysis, it did make full sky line removal impossible. A set of standard spectroscopic star observations were ob-

\footnotetext{
8 Based on observations obtained at the Gemini Observatory, which is operated by the Association of Universities for Research in Astronomy, Inc., under a cooperative agreement with the NSF on behalf of the Gemini partnership: the National Science Foundation (United States), the Science and Technology Facilities Council (United Kingdom), the National Research Council (Canada), CONICYT (Chile), the Australian Research Council (Australia), Ministério da Ciência, Tecnologia e Inovação (Brazil) and Ministerio de Ciencia, Tecnología e Innovación Productiva (Argentina)
}

tained on the same night using the $5^{\prime \prime}$ slit mask, and so we found that slit losses through the $1^{\prime \prime}$ slit mask were negligible.

\subsection{VLA Radio Observations}

We observed NGC4178 on 2012 July 14-15 with the NRAO9 Karl G. Jansky VLA (Perley et al. 2011) while in its B-configuration (program AS1114, 12A-093). In the $1.5 \mathrm{hr}$ observing run, a single 18 min scan of the galaxy was obtained at C-band while two 18 min scans were obtained at X-band. The target scans were bracketed with observations of the point source J1224+0330 for phase calibration while 3C 286 was utilized as the primary flux and bandpass calibrator. After basic calibration in AIPS, we produced CLEAN images at C-band split into two $1 \mathrm{GHz}$ wide side-bands centered at 5.0 and $6.0 \mathrm{GHz}$ with off-source $\mathrm{rms}(1 \sigma)=11.9 \mu \mathrm{Jy} / \mathrm{beam}$ and $12.6 \mu \mathrm{Jy} /$ beam, respectively. The center frequency of the X-band image was $9.0 \mathrm{GHz}(2 \mathrm{GHz}$ bandwidth) with an off-source rms of $6.7 \mu \mathrm{Jy} /$ beam. The image beamsizes were $1.19^{\prime \prime} \times 1.49^{\prime \prime}(5 \mathrm{GHz}), 1.03^{\prime \prime} \times 1.28^{\prime \prime}(6 \mathrm{GHz})$, and $0.66^{\prime \prime} \times 0.84^{\prime \prime}(9 \mathrm{GHz})$.

In the VLA images, diffuse steep-spectrum emission from the NGC4178 galaxy was detected but the Chandra detected nucleus (A) and the three off-nuclear X-ray point sources (B, C, and D) reported in S12 were not detected. For the nucleus, we measured $3 \sigma$ point-source upper limits of $<84.9 \mu \mathrm{Jy}(5 \mathrm{GHz}),<46.8 \mu \mathrm{Jy}(6 \mathrm{GHz})$, and $<24.9 \mu \mathrm{Jy}(9 \mathrm{GHz})$ at the Chandra X-ray position. These values are larger than the off-source rms due to the presence of the NGC4178 galaxy emission. The corresponding limit from our previous analysis of an archival VLA $5 \mathrm{GHz}$ exposure was $<230 \mu \mathrm{Jy}(\mathrm{S} 12)$. For the offnuclear X-ray sources, we measured $(5 \mathrm{GHz}, 6 \mathrm{GHz}, 9$ $\mathrm{GHz})[\mu \mathrm{Jy}]$ upper limits of $(<38.7,<55.2,<25.5)$ for $\mathrm{B},(<27.0,<36.6,<17.1)$ for $\mathrm{C}$, and $(<35.7,<31.2$, $<19.8$ ) for D. The $5 \mathrm{GHz}$ values are $4.4-5.6 \times$ smaller than determined from the archival VLA data (See S12). Note that the diffuse galactic radio emission is resolved in the $\sim$ arcsecond resolution VLA image (Figure 1) and exhibits a clumpy appearance due to the CLEAN processing of the data with limited $(u, v)$ coverage. In this respect, the local peak $(66 \mu \mathrm{Jy} /$ beam $)$ in the new VLA $5 \mathrm{GHz}$ image of the galaxy that we noted in our previous analysis of a sparser archival VLA dataset (S12) is noticeably offset (about $1.7^{\prime \prime}$ ) from the Chandra nucleus and is likely unrelated to the AGN activity.

\section{RESULTS}

The calibrated Gemini spectrum can be seen in Figure 2. Emission line and stellar population fitting were done with simplefit (Tremonti et al. 2004) using the stellar population model of Bruzual \& Charlot (2003). One set of 1-dimensional ( $1^{\prime \prime}$ aperture) and 2-dimensional spectra were produced by performing sky subtraction using the ends of the slit as the sky sample. The line fluxes for the central $1^{\prime \prime}$ are listed in Table 1 .

\subsection{The Dynamical Center}

9 The National Radio Astronomy Observatory is a facility of the National Science Foundation operated under cooperative agreement by Associated Universities, Inc. 


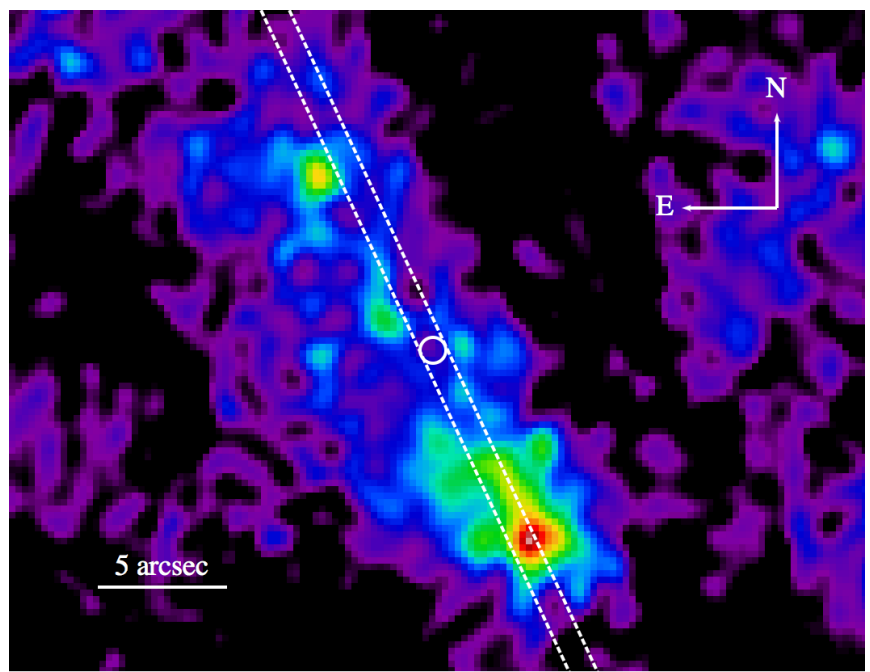

FIG. 1. - VLA $5 \mathrm{GHz}$ image (beam $=1.49^{\prime \prime} \times 1.19^{\prime \prime}$ at position angle $11.2^{\circ}$ ) of NGC 4178 showing diffuse emission from the galaxy. The white circle outlines the Chandra X-ray source, and the dashed white lines indicate the Gemini longslit. $1^{\prime \prime} \simeq 160 \mathrm{pc}$.
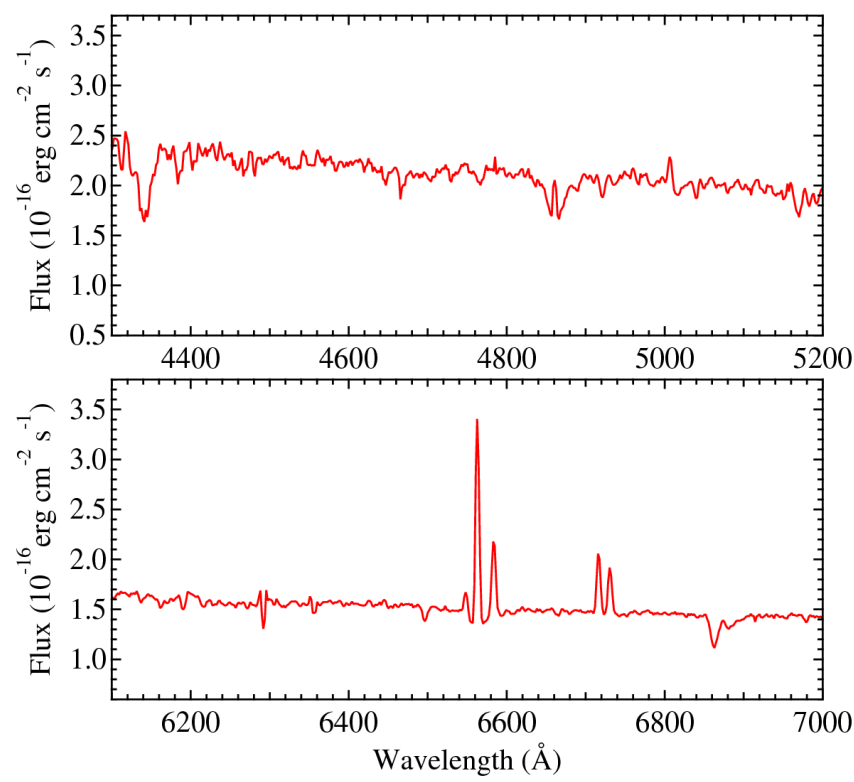

FIG. 2.- The Gemini optical spectrum of the central 1" of NGC 4178 .

TABLE 1

LiNE FLUXES FROM THE CENTRAL $1{ }^{\prime \prime}$

\begin{tabular}{ccc} 
Line & Flux $^{*}$ & $\mathrm{EW}(\AA)$ \\
\hline $\mathrm{H} \beta$ & $0.33 \pm 0.02$ & 1.57 \\
{$[\mathrm{O} \mathrm{III}] \lambda 5007$} & $0.18 \pm 0.02$ & 0.88 \\
$\mathrm{H} \alpha$ & $1.31 \pm 0.01$ & 8.74 \\
{$[\mathrm{~N} \mathrm{II}] \lambda 6584$} & $0.41 \pm 0.01$ & 2.78 \\
{$[\mathrm{~S} \mathrm{II}] \lambda 6717$} & $0.36 \pm 0.01$ & 2.48 \\
{$[\mathrm{~S} \mathrm{II}] \lambda 6731$} & $0.26 \pm 0.01$ & 1.81 \\
\hline
\end{tabular}

* in units of $10^{-15} \mathrm{erg} \mathrm{s}^{-1} \mathrm{~cm}^{-2}$
To derive a robust rotation curve, we measure the radial velocity as a function of radius in two ways: 1) by cross-correlating the spectrum at each spatial position against galaxy templates to determine the velocity, and 2 ) by fitting directly for the centroids of the $\mathrm{H} \alpha$ emission line. For each method, we treat each spatial row of the longslit spectrum independently, but we note that the slit width and seeing are both larger than this pixel size, such that neighboring rows are not independent. For our purposes, it is desirable to assess whether emission line ratios which rise and then fall across a small spatial extent indicate an unresolved AGN source, or arise within a larger resolved region.

Under the first method, we follow a similar procedure to that used by SDSS, where the spectrum at each position is transformed into an evenly spaced grid in log wavelength, and then cross-correlated with several similarly-prepared template spectra to determine the redshift (or velocity) of that spectrum with respect to the rest frame. Under the second method, using the crosscorrelation result as a first-guess, we fit and subtract off the absorption component of each spectrum (following the procedure described below), and then measure the centroid of a Gaussian fit to the $\mathrm{H} \alpha$ line. We note that the two methods yielded consistent curves at all radii for this galaxy.

To the raw measured rotation data, we fit a smooth function of the form $V(R)=V_{\mathrm{MAX}}(R+\Delta R) /((R+$ $\left.\Delta R)^{a}+R_{s}^{a}\right)^{1 / a}+\Delta V$ (Böhm et al. 2004; Moran et al. 2007), where $R$ is the radius, $a$ and $R_{s}$ are free parameters that govern the shape of the rotation curve and its turn-over, $\Delta V$ is the offset of the galaxy's central velocity from the given value $(z=0.001248)$, and $\Delta R$ is the radius offset . Both $\Delta R$ and $\Delta V$ are left free to vary in the fit, but are constrained to stay within a reasonable range of values ( $5^{\prime \prime}$ and $75 \mathrm{~km} \mathrm{~s}^{-1}$, respectively). Notably, we find no evidence that $\Delta R$ is significantly different from zero (the uncertainty, as estimated from bootstrapping, was better than $\sim 2^{\prime \prime}$, or $\sim 320 \mathrm{pc}$ ), implying that the presumed center of the galaxy (the location of the Chandra X-ray source measured in (S12) is also consistent with being its rotational center (Figure 4). While the error on the location of the dynamical center appears to preclude us from completely ruling out the possibility that the dynamical center is coincident with the local radio peak $1.7^{\prime \prime}$ northeast of the Chandra source, there are two reasons why this scenario is unlikely. First, the error in the determination of the dynamical center is along the spatial direction of the slit, which did not cover the local radio peak (See Figure [3), so the velocity offset at the location of the radio peak is not known. Second, the Chandra source is coincident with the Two Micron All Sky Survey photocenter, which is coincident with a nuclear star cluster (NSC, see Section 3 in S12), making it $a$ priori more likely that the dynamical center is coincident with the Chandra X-ray source.

After deriving the best-fit rotation curve, we adjust the spectrum at each spatial position to its rest-frame wavelength scale, and perform all further analysis in the rest frame. 


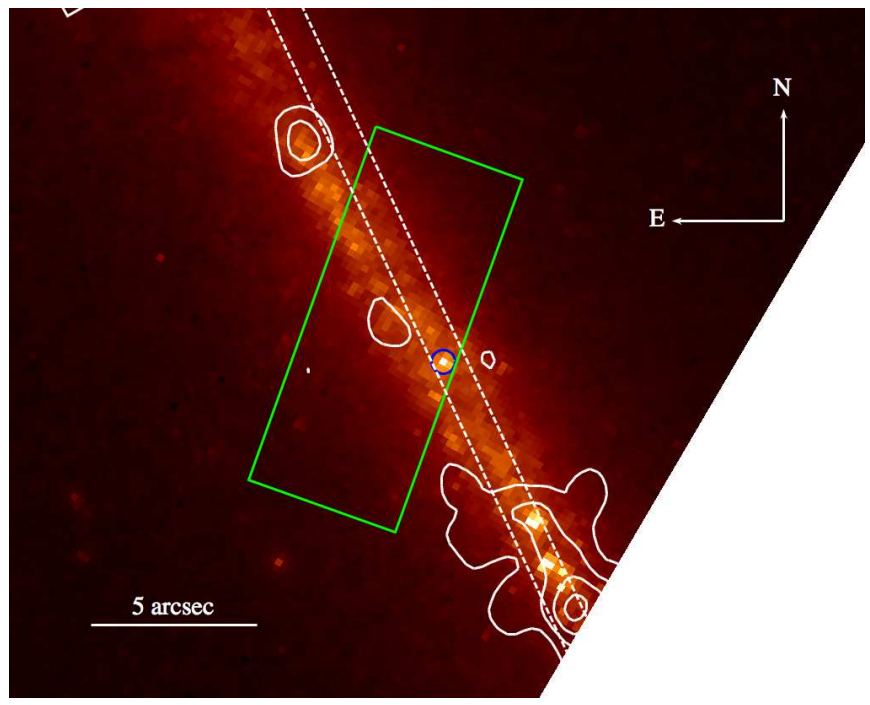

FIG. 3.- The Hubble Space Telescope H-band image of the nuclear region of NGC 4178 with the Spitzer SH slit outlined in green, the Chandra X-ray source outlined in blue, and the Gemin longslit outlined in dashed white. The white contours are the VLA $5 \mathrm{GHz}$ radio contours. $1^{\prime \prime} \simeq 160 \mathrm{pc}$.

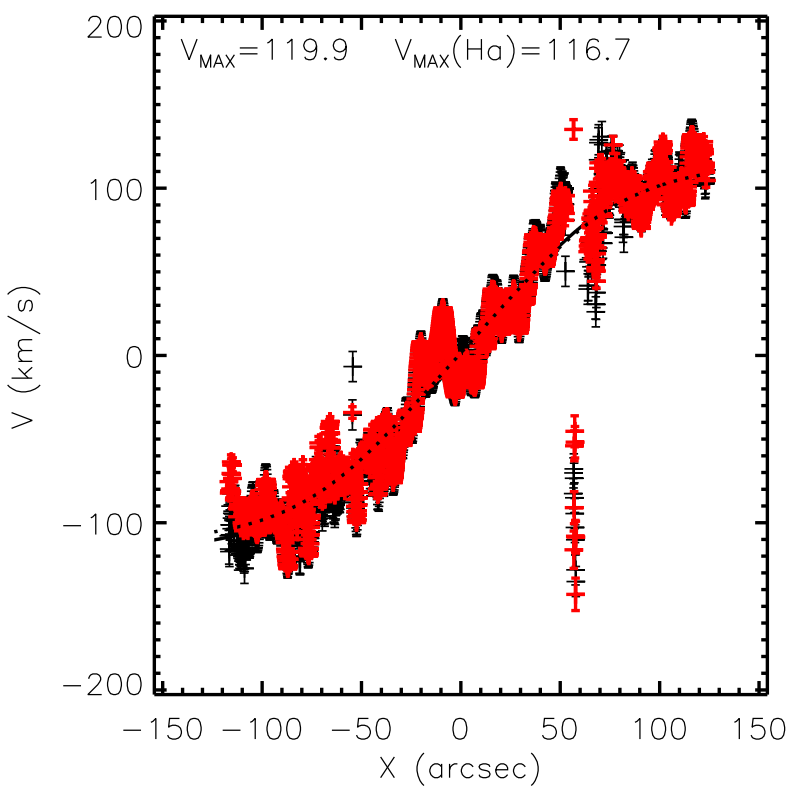

FIG. 4.- The rotation curve of NGC 4178. The red points are the $\mathrm{H} \alpha$, while the black points are from the absorption line fits. The $\mathrm{x}$ axis is defined relative to the Chandra nuclear point source. The uncertainties in the velocities are less than $\sim 10 \%$, or $10-15 \mathrm{~km} \mathrm{~s}^{-1}$ for both $\mathrm{V}_{\mathrm{MAX}}$ and $\mathrm{V}_{\mathrm{MAX}}(\mathrm{H} \alpha) .1^{\prime \prime} \simeq 160 \mathrm{pc}$.

We employ a modified version of the simplefit code that fits stellar templates and emission lines for each row of the 2-dimensional longslit data. In order to increase $\mathrm{S} / \mathrm{N}$, the spectra were extracted in $1^{\prime \prime}$ bins along the spatial direction of the slit, with each consecutive bin shifted by 1 row. This had the effect of slightly smoothing the data and reducing the noise. Each extracted spectrum was fit with a linear combination of templates drawn from the Bruzual \& Charlot (2003) single stellar population models of varying metallicities, masking out the

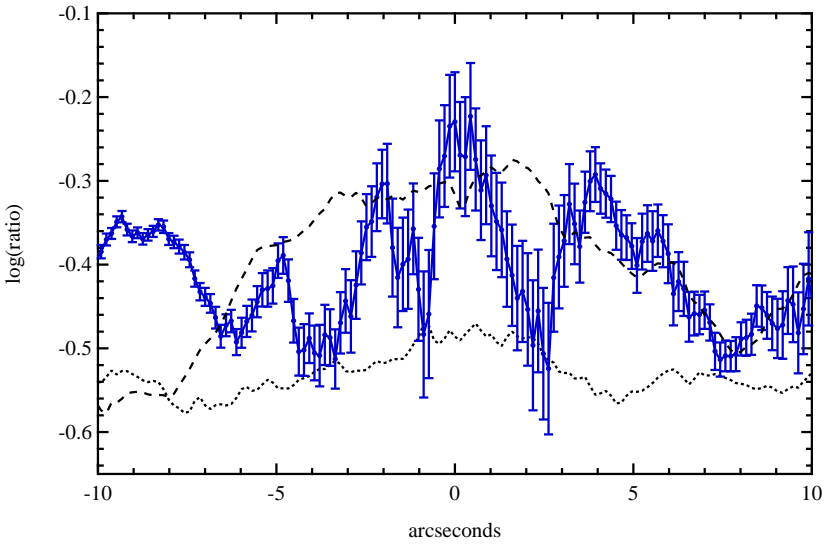

FIG. 5.- BPT diagnostics as a function of distance from the nuclear source $\left(1^{\prime \prime} \simeq 160 \mathrm{pc}\right)$. Blue: $[\mathrm{O} \mathrm{III}] / \mathrm{H} \beta$ with $1 \sigma$ error bars. Dotted: [N II] $/ \mathrm{H} \alpha$. Dashed: $[\mathrm{S}$ II $] / \mathrm{H} \alpha$. The error bars on $[\mathrm{N} \mathrm{II}] / \mathrm{H} \alpha$ and $[\mathrm{S} \mathrm{II}] / \mathrm{H} \alpha$ are not significantly larger than the markers, and so have been omitted for clarity.

emission lines. The best-fitting stellar continuum model was then subtracted from the measured spectrum, creating an emission-line only spectrum where the Balmer emission lines can be measured free of contamination from the underlying stellar continuum. We then fit Gaussian functions to the emission lines, with the widths and centers of the Gaussian free to vary.

In addition to the Balmer lines $\mathrm{H} \alpha$ and $\mathrm{H} \beta$, we also measure the forbidden lines [O III $] \lambda 5007$, $[\mathrm{N}$ II] $\lambda 6548 / 6584$, and $[\mathrm{S} \mathrm{II}] \lambda 6717 / 6731$, which are required $\mathrm{t}$ measure metallicity and test for the presence of an AGN. To assess the presence of an AGN, we use the Kewley et al. (2001) version of the classic Baldwin, Phillips \& Terlovich (1981), or BPT, diagnostic, which utilizes the line flux ratios of $[\mathrm{O}$ III $] \lambda 5007$ to $\mathrm{H} \beta$, [N II] $\lambda 6584$ to $\mathrm{H} \alpha$, and $[\mathrm{S} \mathrm{II}] \lambda 6717 / 6731$ to $\mathrm{H} \alpha$. As a function of radius, we plot these line ratios to assess how rapidly the ionization state of the ISM may change with location in the galaxy Figure 5. While the line ratios vary with radius, at all points their location on the BPT diagram is consistent with originating in HII regions.

\subsection{The Fundamental Plane}

In S12, we used a radio upper limit obtained from archival VLA data combined with the Chandra X-ray detection to set an upper limit on the nuclear black hole's mass using the so-called fundamental plane of black hole activity (Merloni et al. 2003; Falcke et al. 2004; Merloni et al.|2006; Wang et al.|2006; Li et al. 2008; Gültekin et al. 2009; Miller-Jones et al. 2012; Bonchi et al. 2013). With the new $5 \mathrm{GHz} 3 \sigma$ flux density upper limit of $<84.9 \mu \mathrm{Jy}$ and the $0.5-10 \mathrm{keV}$ luminosity calculated from the scenario in which the AGN is heavily embedded with only a few non-Compton thick openings in which X-ray flux escapes (see Section 3.1 of $\mathrm{S12}$ ), we find a predicted black hole mass of $M_{\mathrm{BH}} \lesssim 8.4 \times 10^{4} \mathrm{M}_{\odot}$ using the Miller-Jones et al. (2012) relation $(1 \sigma$ scatter $=0.44 \mathrm{dex})$, which may be more valid than the Gültekin et al. (2009) relation for lower-mass systems because the Gültekin et al. relation was derived for black hole masses greater than $10^{6} \mathrm{M}_{\odot}$ (see Section 4.2 of Miller-Jones et al.). This is a considerably lower upper limit than our previous value of 
$\sim 2 \times 10^{5} \mathrm{M}_{\odot}$.

However, it is important to point out that the Miller-Jones et al. relation was derived from the strongly sub-Eddington $\left(\dot{M} / \dot{M}_{\text {Edd }}<0.01-0.02\right)$ sample of Plotkin et al. (2012), while significant scatter exists for more highly-accreting objects (e.g. Körding et al. 2006), as the AGN in NGC 4178 likely is (S12). We discuss this issue in the next section.

\section{DISCUSSION}

With the confirmation that the dynamical center of NGC 4178 coincides with the location of the Chandra X-ray point source, an important property of the AGN in this galaxy has been confirmed. While bulgehosting galaxies usually have dynamical centers very clearly aligned with their photocenters, bulgeless galaxies, especially bulgeless galaxies with dusty bars such as NGC 4178, have much less obvious centers, and several sources within the bar of NGC 4178 could be considered candidates for the dynamical center, such as one of the two IR-bright lobes detected by Spitzer (see Figure 1 of S12). This analysis highlights the value of using longslit spectroscopy on this unique class of objects, despite the fact that optical emission lines originating from the narrow line region (NLR) may either be too absorbed or too weak to use as an AGN diagnostic by themselves.

It is not clear from the data alone whether it is heavy absorption that prevents NGC 4178 from being optically classified as an AGN or if it is simply that the AGN is too weak to be detected above surrounding star formation. By comparing the X-ray properties of the AGN with the bolometric luminosity predicted from the $[\mathrm{Ne} \mathrm{V}]$ $14.3 \mu \mathrm{m}$ emission, $\mathrm{S} 12$ found that the AGN in NGC 4178 is indeed likely to be heavily obscured, and so we should therefore not be surprised that this galaxy shows no signs of an AGN at optical wavelengths. Even with heavy obscuration, we may be able to detect an increase in ionization parameter near the AGN, giving some clues as to degree of absorption involved. One advantage of longslit spectroscopy is that it has allowed us to examine the ionization state of the ISM as a function of radius from the AGN. Of the three ionization parameters used in Kewlev et al. (2001), all three appear to rise near the galactic center, but only $[\mathrm{O} \mathrm{III}] / \mathrm{H} \beta$ line flux ratio appears to increase sharply enough at the galactic center to possibly be considered local to the AGN (Figure 5). However, the magnitude of the rise in the line flux ratio at the center is comparable to that of the nearby offnuclear variations. The rise in the line flux ratio at the center cannot alone be considered unambiguous evidence for the AGN.

There is, however, some amount of scatter in the $L_{[\mathrm{NeV}]} / L_{\mathrm{bol}}$ relation $(0.53 \mathrm{dex})$, and a portion of this scatter is likely due to $[\mathrm{Ne} \mathrm{V}]$ contamination by heavy starburst activity (e.g. Veilleux et al. 2005). In order to explore this possibility, we plot the star formation rate (SFR) as a function of distance from the nuclear source in Figure 6 using the Kennicutt (1998) relationship between the $\mathrm{H} \alpha$ luminosity and the SFR and using the Charlot \& Fall law (Charlot \& Fall 2000) to empirically correct for extinction assuming optically-thick (Case B) recombination. The IR-bright lobes detected by Spitzer at $\sim 7^{\prime \prime}$ away from the AGN are clearly visible as large increases in the SFR. However, within $\sim 3^{\prime \prime}$ of the nu-

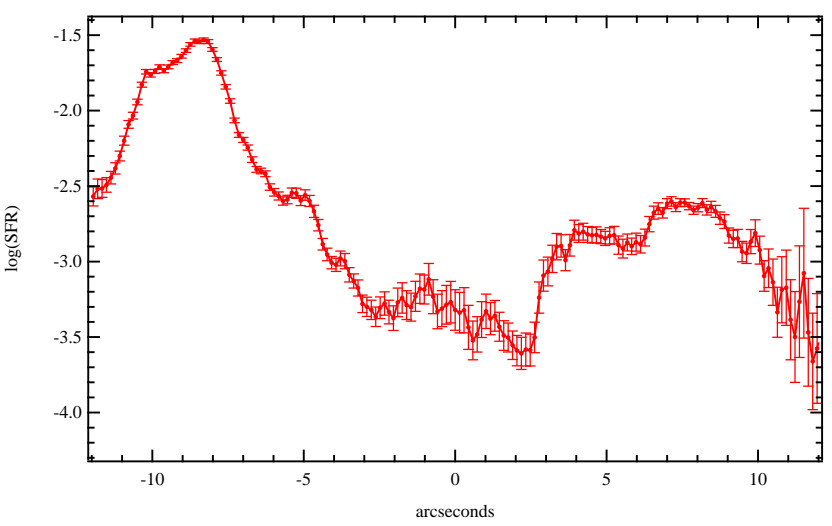

FIG. 6.- $\mathrm{SFR}\left(\mathrm{M}_{\odot} \mathrm{yr}^{-1}\right)$ as a function of angular distance from the nuclear source. Error bars are $1 \sigma .1^{\prime \prime} \simeq 160 \mathrm{pc}$.

cleus, the SFR hovers at around $10^{-3} \mathrm{M}_{\odot} \mathrm{yr}^{-1}$, indicating that there is no significant starburst activity near the nucleus, as is implied by the absence of significant $\mathrm{Pa}-\alpha$ emission noted by S12, Figure 2 therein. Being an extremely late-type, and therefore very dusty galaxy, it is natural to ask if there might be a deeply buried starburst contributing to the $[\mathrm{Ne} \mathrm{V}]$ emission that could be obscured even in the near-IR. To explore this possibility, we used the tight relationship between $\left.L_{[\mathrm{Ne}} \mathrm{II}\right]+[\mathrm{Ne}$ III] and SFR (Ho \& Keto 2007), which is largely insensitive to differences in metallicity, to calculate the SFR at the nucleus of NGC 4178 under the possible scenario in which the $[\mathrm{Ne} \mathrm{II}]+[\mathrm{Ne} \mathrm{III}]$ emission is due to a buried starburst and not to the presence of an AGN. Using the best-fit ionization fraction values of Ho \& Keto, we derive a SFR of $\sim 4 \times 10^{-2} \mathrm{M}_{\odot} \mathrm{yr}^{-1}$. As this number is consistent with the rate predicted by the $\mathrm{H} \alpha$ emission within the margin of error, we conclude that there is not a buried starburst contributing to the [Ne V] emission in NGC 4178.

The new upper limit to the $5 \mathrm{GHz}$ radio luminosity further constrains the upper limit on the black hole mass to within the same order of magnitude as the Eddington lower mass limit implied by the $[\mathrm{Ne} \mathrm{V}]$ luminosity. This would seem to corroborate the scenario of an IMBH deeply embedded in a heavy absorbing material and accreting at a high rate. However, it is important to note that the Chandra point source in NGC 4178 is thus inferred to be in the 'high-soft' state of black hole X-ray emission (see section 3.1 of S12), and so the radio and X-ray luminosities may be only weakly dependent on the mass of the nuclear black hole (for details, see Wang et al. 2006; Li et al. 2008). The upper limit on the mass implied by the deeper VLA measurements should therefore be considered tentative (Although see Gliozzi et al. 2010, for an example of an AGN accreting very close to the Eddington limit with a mass accurately predicted by the fundamental plane).

\section{SUMMARY AND CONCLUSIONS}

We have analyzed Gemini longslit optical spectroscopy of NGC 4178, as well as deeper VLA radio observations. By performing a full spatial spectroscopic characterization of the galaxy, as well as more tightly constraining its radio properties, we have found the following results:

1. The dynamical center of NGC 4178 is coincident with the location of the Chandra X-ray point source 
discovered by S12, providing further support for the presence of an AGN in this galaxy, as well as confirming that this galaxy contains a nuclear star cluster.

2. While the ionization state of the ISM near the nucleus appears to increase, NGC 4178 remains classified as optically normal, further underscoring the need for the penetrative power of mid-IR and Xray observations in finding buried or energetically weak AGNs in this class of galaxy.

3. From new, deeper constraints on the $5 \mathrm{GHz}$ radio luminosity, the upper limit on the nuclear black hole's mass appears to be near the Eddington limit implied by its mid-IR properties, lending support to the notion that this object is deeply embedded and accreting at a high rate. The uncertainty in applying the fundamental plane to highly-accreting black holes, however, means that we should consider this to be a tentative result.

\section{ACKNOWLEDGEMENTS}

We thank the referee for their very thorough review and insightful comments that has significantly improved this paper. N. J. S. and S. S. gratefully acknowledge support by the Chandra Guest Investigator Program under NASA Grant G01-12126X. C. C.C. was supported at NRL by a Karles' Fellowship and NASA DPR S-15633-Y. M. G. acknowledges financial contribution from the agreement ASI-INAF I/009/10/0.

\section{REFERENCES}

Araya Salvo, C., Mathur, S., Ghosh, H., Fiore, F., \& Ferrarese, L. 2012, ApJ, 757, 179

Barth, A., Ho, L., \& Sargent, W. 2002, AJ, 124, 2607 . 2004, in ASP Conf. Ser. 311, AGN Physics with the Sloan Digital Sky Survey, ed. G. T. Richards \& P. B. Hall (San Francisco: ASP), 91

Barth, A. J., Strigari, L. E., Bentz, M. C., Greene, J. E., \& Ho, L. C. 2009, ApJ, 690, 1031

Böhm, A., et al. 2004, A\&A, 420, 97

Bonchi, A., La Franca, F., Melini, G., Bongiorno, A., \& Fiore, F. 2013, MNRAS, 429, 1970

Bonoli, S., Mayer, L., \& Callegari, S. 2012, arXiv:1211:3752

Bruzual, G., \& Charlot, S. 2003, MNRAS, 344, 1000

Charlot, S., \& Fall, S. M. 2000, ApJ, 539, 718

Desroches, L. B., \& Ho, L. C. 2009, ApJ, 690, 267

Dewangen, G. C., Mathur, S., Griffiths, R. E., \& Rao, A. R. 2008, ApJ, 689, 726

Falcke, H., Körding, E., \& Markoff, S. 2004, A\&A, 414, 895

Farrell, S. A., et al. 2012, ApJ, 747, L13

Filippenko, A. V., \& Ho, L. C. 2003, ApJ, 588, L13

Ghosh, H., Mathur, S., Fiore, F., \& Ferrarese, L. 2008, ApJ, 687, 216

Gliozzi, M., Satyapal, S., Erancleous, M., Titarchuk, L., \& Cheung, C. C. 2009, ApJ, 700, 1759

Gliozzi, M., Papadakis, I. E., Grupe, D., et al. 2010, ApJ, 717 1243

Greene, J. E., \& Ho, L. C. 2004, ApJ, 610, 722

Greene, J. E., \& Ho, L. C. 2007, ApJ, 670, 92

Greene, J. E. 2012, Nature Communications, 3, 1304

Gültekin, K., et al. 2009, ApJ, 706, 404

Ho, L. C., Filippenko, A. V., \& Sargent, W. L. 1995, ApJS, 98, 477

Ho, L. C., \& Keto, E. 2007, ApJ, 658, 314

Izotov, Y. I., Thuan, T. X., \& Guseva, N. G. 2007, ApJ, 671, 1297

Izotov, Y. I., \& Thuan, T. X. 2008, ApJ, 687, 133

Izotov, Y. I., et al. 2010, A\&A, 517, 90

Jiang, Y., Greene, J. E., \& Ho, L. C. 2011, ApJ, 737L, 45

Jiang, Y., Greene, J. E., Ho, L. C., Xiao, T, \& Barth, A. J. 2011, ApJ, 742, 68J

Kennicutt, R. C. 1998, ApJ, 498, 541

Kewley, L. J., Heisler, C. A., Dopita, M. A., \& Lumsden, S. 2001, ApJS, 132, 37
Körding, E., Falcke, H., \& Corbel, S. 2006, A\&A, 456, 439 Kormendy, J., \& Kennicutt, R. C. 2004, ARA\&A, 42, 603

Li, Z., Wu, X., \& Wang, R. 2008, ApJ, 688, 826

Mathur, S., Ghosh, H., Ferrarese, L., \& Fabrizio, F. 2008, AIPC, 1053, 43

McAlpine, W., Satyapal, S., Gliozzi, M., et al. 2010, ApJ, 728, 1

Merloni, A., Heinz, S., \& Di Matteo, T. 2003, MNRAS, 345, 1057

Merloni, A., Körding, E., Heinz, S., Markoff, S., Di Matteo, T., \& Falcke, H. 2006, New Astron., 11, 567

Miller-Jones, J. C. A., et al. 2012, ApJ, 755, 1

Moran, S. M., Miller, N., Treu, T., Ellis, R. S., \& Smith, G. P. 2007, ApJ, 659, 1138

Nucita, A. A., Manni, L., De Paolis, F., Vetrugno, D., \& Ingrosso, G. 2013, A\&A, 550, 18

Perley, R. A., Chandler, C. J., Butler, B. J., \& Wrobel, J. M. 2011, ApJ, 739, L1

Plotkin, R. M., Markoff, S., Kelly, B. C., Körding, E., \& Anderson, S. F. 2012, MNRAS, 419, 267

Reines, A. E., Sivakoff, G. R., Johnson, K. E., \& Brogan, C. L. 2011, Nature, 470, 66

Satyapal, S.,Vega, D., Heckman, T., OHalloran, B., \& Dudik, R. 2007,ApJ, 663, L9

Satyapal, S., Vega, D., Dudik, R. P., Abel, N. P., \& Heckman, T. 2008, ApJ, 677, 926

Satyapal, S., Böker, T., McAlpine, W., et al. 2009, ApJ, 704, 439

Secrest, N. J., Satyapal, S., Gliozzi, M., et al. 2012, ApJ, 753, 38

Shields, J. C., Walcher, J. C., Böker, T., \& Ho, L. C. 2008, ApJ, 682,104

Simmons, B. D., et al. 2013, MNRAS, 429, 2199

Soria, R., et al. 2010, MNRAS, 405, 870

Tremonti, C. A., et al. 2004, ApJ, 613, 898

van Dokkum, P. G. 2001, PASP, 113, 1420

van Wassenhove, S., Volonteri, M., Walker, M. G., \& Gair, J. R. 2010, MNRAS, 408, 1139

Veilleux, S., Cecil, G., \& Bland-Hawthorn, J. 2005, ARA\&A, 43, 769

Wang, R., Wu, X.-B., \& Kong, M.-Z. 2006, ApJ, 645, 890

Webb, N., et al. 2012, Science, 337, 554 